\title{
Understanding Complex Material Systems using Multiple Characterization Techniques
}

\author{
Swarnagowri Addepalli, T. Shalini \\ GE Global Research, Bangalore, India
}

Industrial components are extremely complex and contain several dissimilar and possibly highly incompatible materials that are placed in close proximity to each other, in order to achieve the desired properties in an end application. Examples are metal-ceramic interfaces, and multilayer coatings - to name a few. This could lead to instability and degradation-related issues under the conditions for the end application - such as inter-diffusion, interfacial reactions, delamination and so on. In some situations, in contrast, such changes might result in enhanced stability.

This talk showcases several examples highlighting the significance of using multiple analytical methods in characterization of complex material systems consisting of a combination of oxides, ceramics, coatings, polymers, metals and alloys. Scanning Electron Microscopy (SEM) and Backscattered Electron Imaging (BEI) have been used with elemental composition analysis and/or mapping in Energy Dispersive X-ray spectroscopy (EDX) and Wavelength Dispersive X-ray Spectroscopy (WDS). In addition, X-Ray Diffraction (XRD) and X-ray Photoelectron Spectroscopy (XPS) have been used wherever relevant. The results have been used to identify impurity phases, understand the composition of materials used in high temperature and lighting applications, as well as for elucidating interfacial interactions as well as degradation/failure and erosion/corrosion mechanisms in various material systems.

A few examples from the presentation are given below:

Figure 1 depicts a case study where elemental composition mapping in EDX was used to identify an impurity phase formed during the oxidation of an alloy, which could not be detected using X-ray diffraction analysis, primarily due to its relatively low concentration. XRD analysis of the alloy (Figure 1; left) shows the presence of its various oxides, while EDX composition mapping (Figure 1; right) shows the presence of an impurity phase consisting of zinc, calcium and sulfur, in addition to the oxide formed on the alloy. Based on the distribution of these impurities in the composition maps, it is believed that zinc oxide and calcium sulfate are present in the surface of the oxidized alloy.

Figure 2 shows the thermal stability study of a multilayer coating with a composition $\mathrm{Si} / \mathrm{Cr} / \mathrm{In} / \mathrm{Au}$ that was formed using physical vapor deposition. The as-deposited multilayer coating reveals islands pointing to either a Volmer-Weber (VW) thin film growth mechanism in which the deposited layer (in this case, $\mathrm{Au}$ ) forms 3-dimensional islands in the surface of the substrate, in order to minimize interface energy as well as its surface energy, or the Stranski-Krastanov (SK) growth mechanism, wherein the first layer of deposited atoms wet the surface, but subsequent layers do not. Due to poor chemical affinity between the atoms at the interfaces of the different layers, multilayer coatings that exhibit either the VW or SK growth mechanisms tend to "dewet" upon annealing. Heating the sample from 170 $330^{\circ} \mathrm{C}$ in an inert ambient led to the formation of discrete islands. EDX spectra indicated intense peaks corresponding to Indium in the islands (A), compared to the regions between the islands (B). Upon annealing to $400^{\circ} \mathrm{C}$, the discrete islands formed in the temperature range from $170-330^{\circ} \mathrm{C}$ appear to "spread out", and the islands formed are smaller in size compared to those observed in the SEM images 
of the as-deposited sample. Based on the analyses from SEM/EDX and XPS analyses (not shown), the destabilization mechanism of the $\mathrm{Si} / \mathrm{Cr} / \mathrm{In} / \mathrm{Au}$ multilayer coating was found to be a combination of (a) dewetting of $\mathrm{Au}$ from In at $170-330^{\circ} \mathrm{C}$, or encapsulation of $\mathrm{Au}$ atoms by In, (b) oxidation of Indium and (c) likely formation of a layer containing a mixture of In and $\mathrm{Au}$ atoms ( $\mathrm{Si} / \mathrm{Cr} / \mathrm{In} / \mathrm{In}-\mathrm{Au})$, or a sandwich structure of the type $\mathrm{Si} / \mathrm{Cr} / \mathrm{In} / \mathrm{Au} / \mathrm{In}$ at $400^{\circ} \mathrm{C}$.
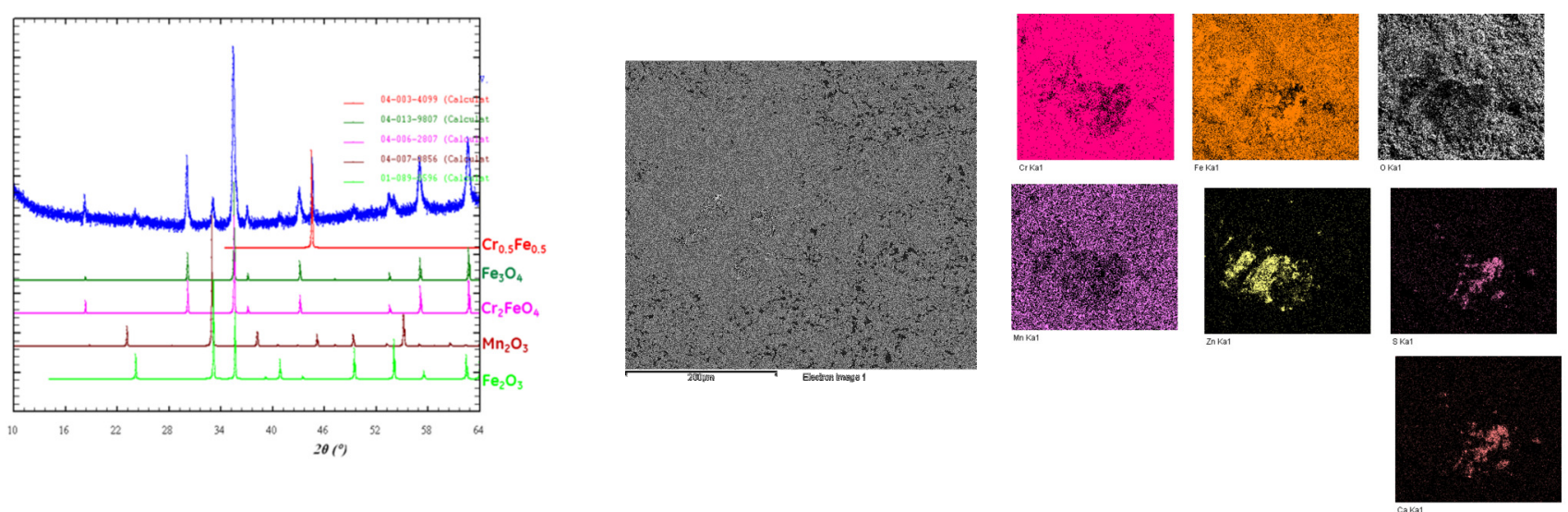

Figure 1. Left - XRD analysis of an alloy, revealing no impurity phases. Right - EDX Elemental composition mapping of the alloy revealing the presence of an impurity phase possibly corresponding to a mixture of zinc oxide and calcium sulfate.
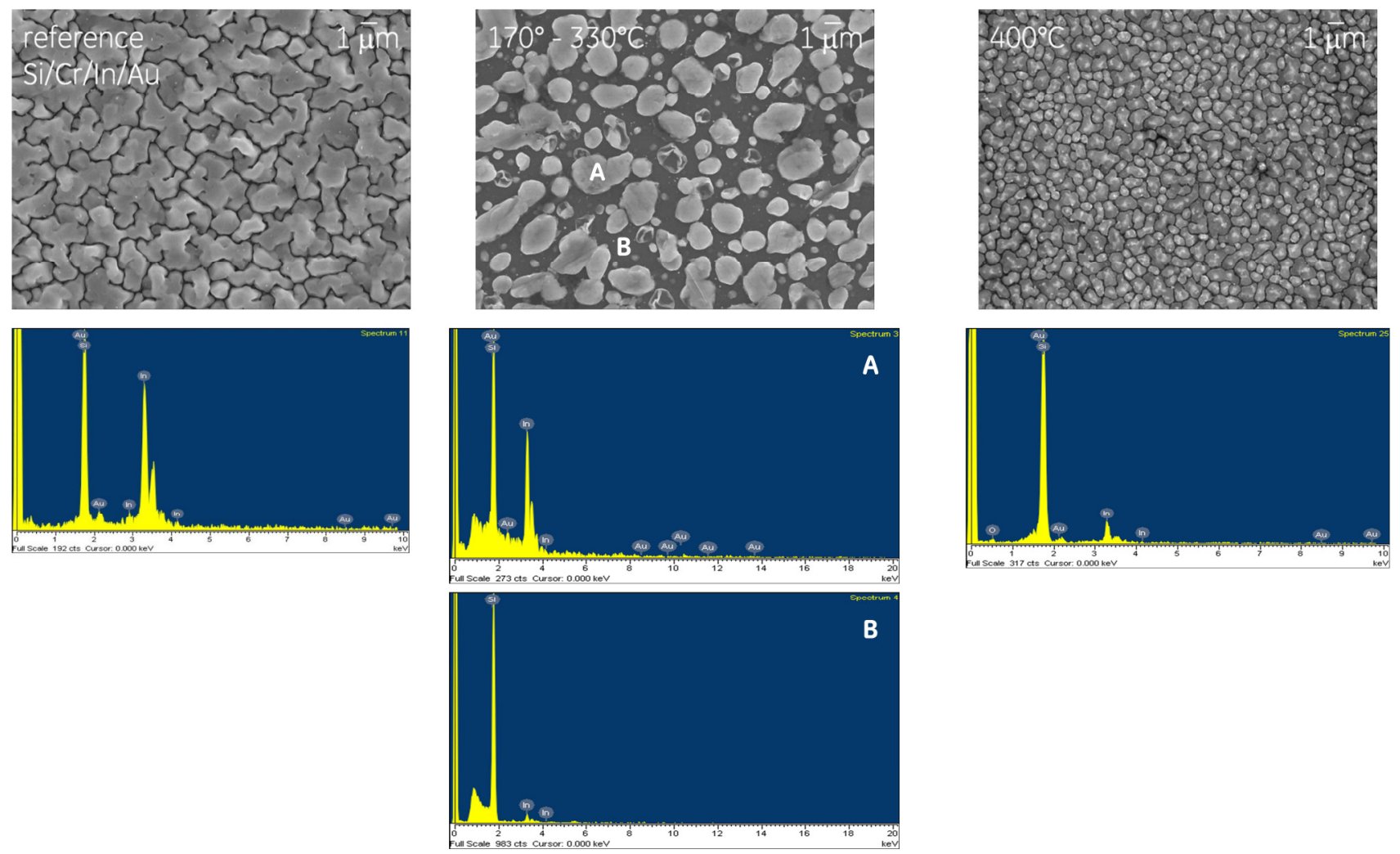

Figure 2. Thermal stability study of an $\mathrm{Si} / \mathrm{Cr} / \mathrm{In} / \mathrm{Au}$ multilayer coating 